NEUES ZUR ÜBERLIEFERUNG DES EVANGELIUMS NICODEMI. 329

gliede dieses geschlechtes auch den stifter unseres grabsteines sehen, vielleicht sogar dessen stiftung mit der stiftung der messe zeitlich in verbindung bringen dürften: aber wir haben keinen anlass, solches zu schliessen. Die Holzschuher dürfen uns nur ein beispiel für viele sein; ebensogut wie sie können auch andere Nürnberger familien in beziehungen zu Eschenbach gestanden haben, ist doch auch noch eine zweite frühmesse daselbst wenig später, im jahre 1410, durch die Nürnberger bürger Eckhardt Neydung und seinen sohn gestiftet worden (vgl. Dumm s. 15). In dieser bürgerschaft Nürnbergs hat aber gewiss auch im 14. jh. schon der meistergesang freunde und pfleger gefunden, wenn auch die blüte der dortigen singschule erst ins 15. und 16. jh. fällt. Wir werden deshalb kaum fehl gehen, wenn wir annehmen, dass aus diesen kreisen der oder die 'verehrer' Wolframs stammten, die - ohne viel von ihm zu wissen - sein andenken als das eines 'meistersingers' durch stiftung eines grabsteins ehren wollten.

(Regesta X 50.145. 150), sowie eine aus dem jahre 1381 (Jahresbericht des hist. vereins für Mittelfranken XX10). Bestätigung der stiftung durch bischof Friedrich IV von Eichstätt erfolgte im jahre 1390; siehe auch Dumm a. a. o. s. 14 .

GIESSEN, den 18. dec. 1908.

\title{
NEUES ZUR ÜBERLIEFERUNG DES EVANGELIUMS NICODEMI VON HEINRICH VON HESLER.
}

Das im besitz von F. Menčik in Wien befindliche, von E. Schröder, Zs. fda. 50, 386 ff. publicierte fragment des Evangeliums Nicodemi kann, wie schon Schröder a.a.o. feststellt, zur textkritik nichts beitragen. Trotzdem ist es in mehrfacher hinsicht nicht uninteressant. Das fragment, nenmen wir es, da es wol überhaupt nur die Veronikalegende enthielt, $W^{1}{ }^{1}$ ),

1) Die bezeichnung $W$ ist schon für die hs. no. 19681 der. Wiener hofbibliothek in anspruch genommen. 
kann in seinem verhältnis zu den anderen hss. genauer bestimmt werden, und zwingt uns, die stellung von $G$ innerhalb des handschriftenschemas, wie ich es in der einleitung zu meiner ausgabe des Ev. Nic.s. xIx aufgestellt habe, etwas zu modificieren.

Dass Wv der von mir mit $\mathbf{z}^{1}$ bezeichneten handschriftengruppe zugehört, ergibt sich mit zwingender notwendigkeit aus den versen $4649 \mathrm{f}$, in denen es den fehler dieser gruppe hiez... schrien gegen liez in Syrien teilt. Aber auch innerhalb dieser gruppe ist sein platz bestimmter anzugeben. Ueberliefert ist die in $\mathrm{Wv}$ enthaltene partie $4530-4658$ in $\mathrm{G}$ und $\mathrm{s}$, zum teil auch in $\mathrm{m}$; die anderen hss. der gruppe reichen nicht so weit. $\left.{ }^{2}\right) \mathrm{Zu}$ keiner der genannten hss. stimmt Wv genau das ist schon durch die nachlässige textbehandlung ausgeschlossen -, aber einige lesarten stehen doch jenen von $G$ sehr nahe.

Am wichtigsten sind die verse $4543 \mathrm{ff}$. Sie lauten im original:

wen die sint blint, haltz und toub

und sint niht wan der sele roub;

4545 wer hier an sie geloubet

der wirt dort beroubet

des ewigen heiles.

Die hs. $s$ hat v. 4544 ausgelassen, die übrigen verse aber ziemlich ungeändert beibehalten; dadurch und durch $G 4544$ wird die ganze stelle für $z^{1}$ gesichert. $G$ hat nun aber in der weise geändert, dass es 4543 in zwei verse auseinander reisst und dafür $4545 \mathrm{f}$. unter gleichzeitiger änderung des wortlautes in einen zusammenzieht. Die stelle lautet dann:

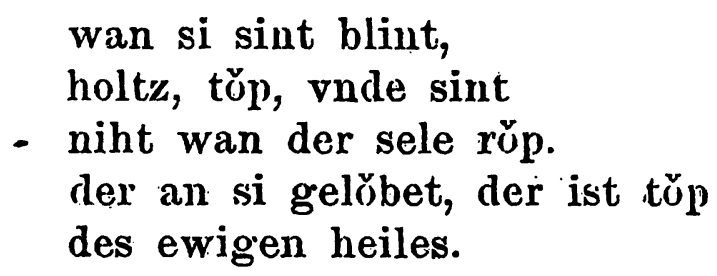

Die für Wv nach ergänzung der lücken anzusetzende fassung:

1) Bibliothek des Lit. vereins no. 224 (1902).

2) Auch keine hs. der weiteren guupe $\mathrm{n}$ ! Ich bemerke das ausdriicklich, weil in meiner ausgabe s. xvin infolge eines druckfehlers irrtïmlich die verse $4 \check{1} 19-4546$ unter dem bestand voin $\mathbf{M}$ erscheinen; gemeint sind die verse $4419-4446$. 
NEUES ZUR ÜBLRLIEFERUNG DES EVANGELIUMS NICODEMI.

wan si sint der sele raup,

der an si gelaubt der ist taup

des evigen hailez

setzt direct die version von $G$ in der vorlage voraus.

Ebenso passen $z u$ $G$ sehr gut die folgenden einzelnen verse, die ich jeweils mit den entsprechenden der hs. G und des originals $(O)$ zusammenstelle.

$\begin{array}{ll}\text { v. } 4570 \text { daz auch si des sellen gelauben warm } & \text { Wv } \\ \text { daz si es geloubell weren G } \\ \text { daz si geloubic weren O und s! } \\ \text { vit tyto seine svn reite W W } \\ \text { mit sînem sun rîten G } \\ \text { mit sinem sone Titen O und s! } \\ \text { v. } 4654 \text { untz si die inneren aessen Wv } \\ \text { untz sich di inren aezzen G } \\ \text { biz sie sich selben ezen O und s! }\end{array}$

Auch v. 4600 ez nah im do chunich wart stimmt zu G; denn das unvollständige anfangswort des verses ist sicher entsprechend der lesart von $G$ (gahes) zu gahez zu ergänzen. Das $z$ kann keine schwierigkeit machen, denn $z$ für $s$ ist in Wv überhaupt häufig.

Die aufgezählten übereinstimmungen zwischen $\mathrm{Wv}$ und $\mathrm{G}$ könnten ihre erklärung darin finden, dass der compilator von Wv die hs. G direct benutzt hat. Dagegen scheint aber anderes zu sprechen. In einigen versen hat $W v$ nämlich die von $G$ aufgegebene lesart des originals beibehalten oder steht diesem wenigstens näher. Ich nenne zuerst v. $4636 v \bar{v}$ dir trevlich diensthaft wesē $\mathrm{Wv}$, und dir dienesthaft wesen $\mathrm{O}$ gegen $v \bar{n}$ dir dienende wescn G. ${ }^{1}$ ) Diese stelle ist allerdings nicht beweisend; denn es wäre immerhin möglich, dass ein schreiber ein in seiner vorlage stehendes dienende selbständig in dienesthaft geändert hätte. Dann wäre die übereinstimmung mit dem original secundär und zufällig.

Wichtig ist dagegen v. 4624 ist nv also betagt Wv, ez was nu ouch also getaget $\mathrm{O}(\mathrm{s})$ gegen ez stet im also bereit $\mathrm{G}$.

In v. 4619 steht $W v$ zwischen dem original und $G$; man vergleiche: du bist an sine stat irkorn $\mathrm{O}$, du pist an des reiches stat erch[oren WV und du bist an des riches rat erchorn G (s schreibt statt dessen du bist zu keiser erchoren!).

1) s hat vollständig geändert: leben | unnl wil mich dinen iuulden geben. 
Dies scheinbare schwanken zwischen $G$ und dem original kann zweierlei ursachen haben. Hat der compilator $G$ direct benutzt, so muss ihm daneben eine zweite dem original näher stehende hs. vorgelegen haben, der er die von $\mathrm{G}$ abweichenden stellen entnahm. Das ist aber bei einem manne, dem es gar nicht auf die qualität seines textes ankam, recht unwahrscheinlich; nur wenn er das bestreben gehabt hätte, einen guten text zu bieten, wäre es erklärlich, dass er sich bei einer zweiten hs. rat geholt hätte. Wir müssen uns also nach einer anderen erklärungsmöglichkeit ümsehen: es kann nur die sein, dass der schreiber von $W v$ nicht nach $G$ selbst gearbeitet hat, sondern nach einer hs., auf die auch G zurïckgeht. Diese vorlage kann aber nicht die hs. $\mathbf{z}^{1}$ gewesen sein, weil $\mathbf{z}^{1}$, wie wir gesehen haben, die stelle $4543 \mathrm{ff}$. noch in der richtigen fassung hatte. Wir müssen also zwischen $z^{1}$ und $G$ noch eine weitere hs. einschieben, der $G$ and $\overline{W v}$ mit verschiedener treue folgten. Ich nenne sie $\mathbf{z}^{4}$; das schema der hss.-gruppe $\mathbf{z}^{1}$ stellt sich darnach folgendermassen dar:

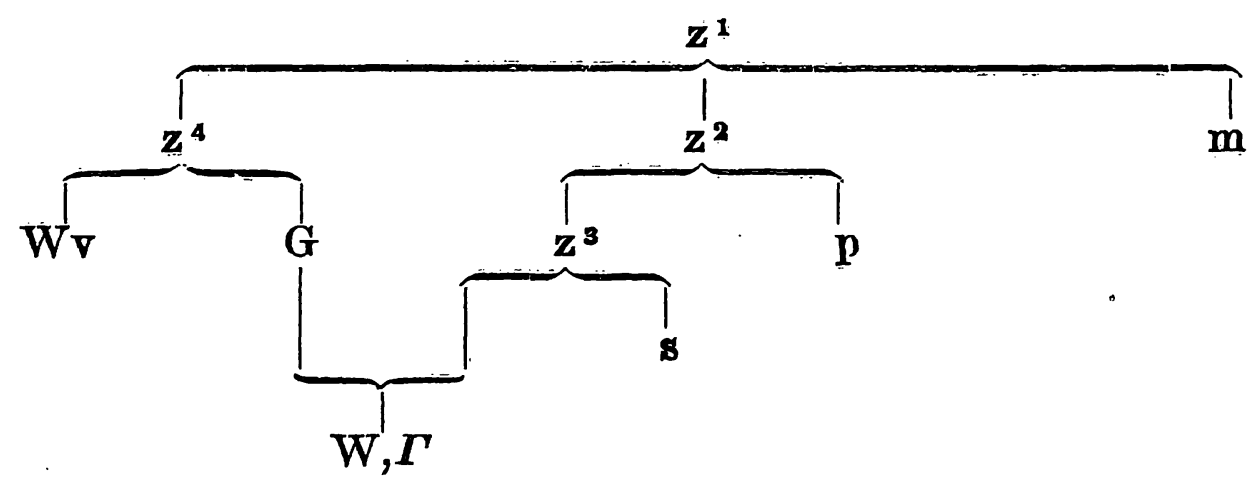

Diese hs. $\mathrm{z}^{4}$ muss in den versen 4543 ff. 4570.4652 und 4654 bereits den uns aus $G$ bekannten feller gehabt haben, sie hatte dagegen in v. 4624, vielleicht auch v. 4636, noch die lesart des originals: In v. 4619 liegen die dinge anders. Hier hat auch der schreiber von $s$ geändert und zu dieser änderung hätte er keine veranlsssung gehabt, wenn in seiner vorlage noch die klare lesart des originals an sine stat gestanden hätte. Umgekehrt wäre die lesart von $G$ und Wv unverständlich, wenn schon in $\mathbf{z}^{1}$ wie in $\mathrm{s}$ ze keiser erchorn ${ }^{1}$ ) gestanden

1) Auch m schreibt: zeinem keiser erwelt. Es ist daraus nicht ohne weiteres auf nähere zugehörigkeit zu s zu schliessen, da es ja sehr nahe lag, an stelle einer unverständlichen lesart dieșe wendung einzusetzen. 
NEUGS ZUR ÜBERLIEFERUNG DIS EVANGELIUMS NICODEMI. 333

hätte. Wir müssen also annehmen, dass bereits der schreiber von $\mathbf{z}^{1}$ statt an sine stat versehentlich an des riches stat schrieb. Diese lesart ist dann in $\mathbf{z}^{4}$ übergegangen, von wo sie unser compilator übernahm, während der schreiber von $G$ einen besserungsversuch machte.

Dass wir auf diese weise zu den früher erschlossenen sechs verlorenen hss. des Evangeliums Nicodemi ( $\left.x, y, z, z^{1}, z^{2}, z^{3}\right)$ nun noch eine siebente erhalten, darf uns nicht stören; denn die zahl der verlorenen hss. hat damit noch nicht die der erhaltenen hss. erreicht, und ich glaube, wir müssen bei allen dichtungen des mittelalters mit einem sehr viel ungünstigeren verhältnis zwischen verlorenen und erhaltenen hss. rechnen und ganz besonders bei einem werk wie dem unsern. Wer kann uns sagen, wie viele hss. im ordensland in den stürmen des 14. und 15. jh.'s vernichtet worden sind! Auch die beliebtheit des im Ev. Nic. dargestellten stoffes spricht für eine reiche handschriftlirhe verbreitung; und für diese beliebtheit ist auch diese neugefundene hs. ein beredter zeuge.

Es ist bisher schon als sicher angenommen worden, dass Menčiks handschrift nicht etwa die beiden gedichte: Christi hort von Gundacher von Judenburg und Heslers Ev. Nic. getrennt enthielt, sondern eine contaminationsarbeit, woran ja auch Schröder nicht zweifelt. Die nahe berührung im inhalt legt das schon sehr nahe; es lässt sich aber auch direct beweisen. Schon Schröder bemerkt, dass der text des ersten blattes von dem sonst bekannten text Gundachers stark abweicht, und besonders stark wird die differenz am schlusse. Dies erklärt sich sehr einfach daraus, dass eben auch hier zum teil schon verse aus dem Ev. Nic. vorliegen. Ich stelle die letzten verse von blatt $1^{\nabla}$ den entsprechenden versen Heslers in paralleldruck gegenüber und hebe das gemeinsame durch sperrung hervor:

Wv (1 $\mathbf{v})$

Si sagten im wie is waz ergangen ir geschiht $\nabla n t z$ en ende a we diser grozsen miszwende $\operatorname{sp}^{\mathrm{a}} \mathrm{ch} \mathrm{d}^{\prime}$ chaiser tybsius

nv mi̊zich imms quuelen sus mit uoten die ich leiden můz.
EvN.

und sageten ime umme Crist die rede wenz anz ende. «O wach der missewende!» sprach der konic Tiberius 4470

«nu muzich immer quelen sus mit disen noten die ich han! 
334 HELM, NEUES ZUR ÜBERLIEFERUNG DES EVANG. NICODEMI.

$$
\mathrm{W} \nabla\left(1^{\mathrm{v}}\right)
$$

Ev.N.

Seit mir $d^{s}$ iesus niht macht půz

ach wie sol iz mir ergan.

(Gundacher v. 4976)

Habt aws ir pylatē iht getan

Hat ir Pilate iht getan?》

Hier können wir deutlich sehen, wie beide gedichte ineinander gearbeitet worden sind. Zwischen dem letzten auf blatt 1 und dem ersten auf blatt 2 stehenden vers aus dem Ev. Nic. liegen 60 verse; so viel konnte die abgeschnittene spalte $1 \mathrm{~d}$ nicht enthalten; der inhalt muss also wesentlich gekürzt worden sein. Bestätigt wird aber auch dadurch wider Schröders annahme, dass beide blätter reste desselben doppelblattes, des innersten einer lage, sind.

Nur mit einem wort sei noch darauf hingewiesen, dass wir in diesem fragment nun bereits den dritten fall einer. compilation des Evang. Nic. mit einer anderen dichtung besitzen. Die beiden anderen compilationen liegen vor in $\mathrm{m}$ (Heinrichs von München fortsetzung der Weltchronik Rudolfs von Ems), wo die verse des Ev. Nic. mit solchen aus dem Passional vermengt sind, und in der hs. W, in welcher das Ev. Nic. mit Bruder Philipps Marienleben zusammengearbeitet ist (vgl. meine einleitung s. XII und xIx).

GIESSEN, den 27. januar 1909.

KARL HELM. 